\title{
DE PLANTIS VENENATIS
}

VENIA. EXP. FACULT. MED. UPSAL.

\section{PRASIDE}
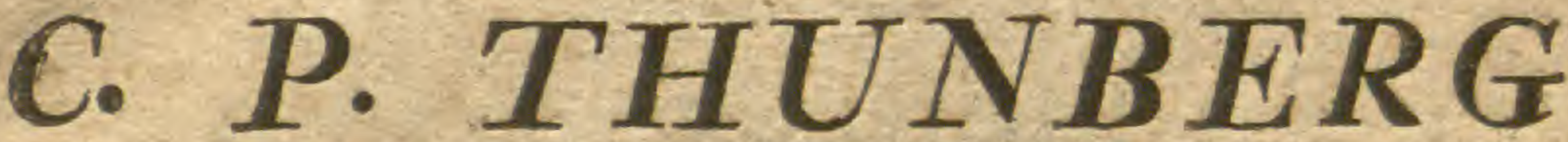

Commendatore Reg, Ord. de WAsa

Med. Doct. Prof. Med, et Bot. Reg, et Ord. Reg. Colt. Santt. Mema: Hon. Acad. Gasar. Petrorol, ex Nat, Curios, Rgg. Scisnt, Ups. Lond. Holm, Agrtc. Med. et Evang. Socigt, Oecon. upsaz. Patr. Holm, zt. Civ.Oecon. Finz. Honor. Wermel. Junec. Westm. Calm. et Órebr. Berol et lips. Nat. Scrut. Paris, Hafn. Moscou, ex Haleng. Hist. Nat. Gorenir. Philad, Lund, Harlem. Amsteld. Oecon, ex Inst. Lit t. Ze-

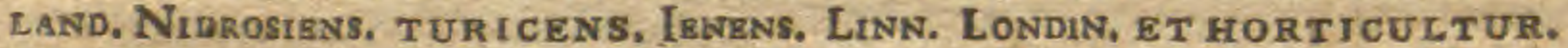
Reg. Seignt. Phys. et Phytograpi, Götringens. Scient, Gothob. Monach. Ertang. Wetter. Nanctins, Marpurg. Med. Paris. Emur. iet Linn. Monspad. Med. Matritens. Med, zt Nat, Studios, Emimaurg. E? Petrop. Medico Chir. et pharmac. Membro nec non Acad, Scignz. Paris, et Institutnation. Monspgligns. Agricult. Paris. Mrdic. Lombut.

Scandinav. Florentin, et Bntavin, Ind, Or, Corkegrond。

PRO GRADU MEDICO

\section{P. P. \\ NICOL. JOH. LJUNGBERG}

Stip. Rog. Smolandus,

IN AUDIT. BOT. DIE XXII MAJI MDCCCXXII

HORIS CENSUETIS

P. 1. -7 :

Mo. Bot Garden,

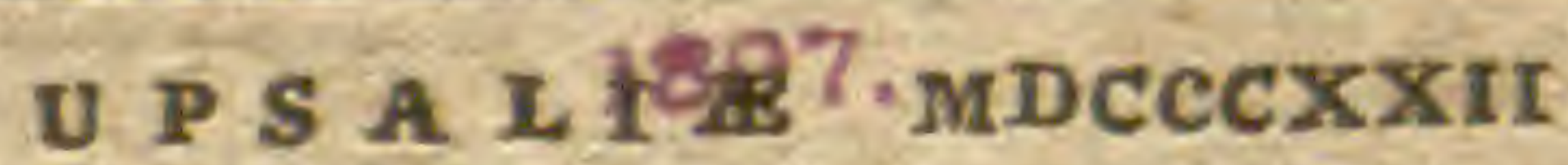

EXCUDIBANT IRGI ACADEMII TYPOGRÁPHI 
MAXIMLE REVERENDIS AC PRACLARISSIMIS DOMINIS MAG. JONAE LJUNGBERG PASTOR, \& PRAPOSIT, IN FRYELED \& HAGSHULT

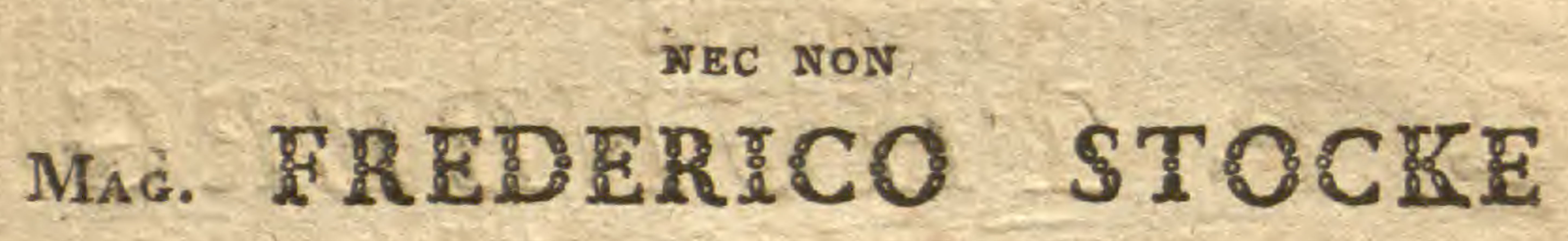
PASTOR. IN JARSTORP \& BANKERYD

\section{Patruis Optimis}

SACRUM

voluit, debuit N. J. LJUNGBERG. 


\section{DE PLANTIS VENENATIS}

\section{W}

eneni idea tam ampla quancoque patet, ut non facile fit dicto, quid fir venenum? Lantimo enim fenfu comprehentit morbos exanthematioos ornes "t niorfuras animalium, qua non raro morrem comitem habent. A nonntllis quoque huc referuntur vacores aërei, qui pulinopibus fumsopere noxit momento fape chins vitæanimalis fcintulam exttingvunt. Latiori feniu omnia remedia, falutifera licet, venena fuerunt appellara; ftrictiori autem fenfu ea tantum aucrores nonnuli fic denominata voluerunt, que minima dofi vehementisfime agunt, vel nexum corporis folvunt et deftruunt, fympromata morbofa graviora excitant et vel ipfam mortem inferunt.

Definitione itaque Logica non adeo facile determina. lür genuina notio veneni.

$$
\text { §. } 2 .
$$

Sapientiores Medici in eo facile omnes conveniunt: yuods

1:0 Nutriens dicendum fit omne id, quod in corpus ingeftum et vegetabilis vita viribus disfolutum, in naturam

Mo. Bot. Garden. 
ejus, absque ullo incommoto asfimilari poteft, in infantia et juventute ad incrementum corporis et dein per totum vita curriculum ad ejus nutrimentum conferre valet. Quod

2:0 Medisamina appellari mereantur omnes illæ materiæ, quxe vel fimplices, vel compofitx, vel artificiofe præparatæ, ftatum corporis morbofum mutant et corrigunt, adeoque amisfam valetudinem reftituunt. Quodque

3:0 Venena nominari debeant tantum illa, ex remediorum alias clasfe defumta, fimplicia vel præparata, qua minori dofi vehementisfima excitant fymptomata, ur vo. mitum, diarrhoeas, colicas, inflammationes cum infequenre gangræna et morte; vel turbas vitæ animalis periculi plenas causfant, ut lipothymiam, lethargum, apoplexiam.

\section{§. 3 .}

Edulia omnia, quæ dentibus contrita et cum saliva ac liquore gaftrico mixta ope morus inteftinorum periftaltico, e regno animali in gelatinam tenuem, e regro vegetabili in emulfionem disfolvuntur, dulcia utplurimum et glutinofa funt. Omnia itaque dulcia mite non modo nutriunt, fed etiam pingvefaciunt; et farinofa omnia, guo uberiori abundant glutine, eo magis quoque nutrire folent. Hæc in corpore fano nullas excitant rurbas, dolores nullos. Tenuisfima particulie disfolutæ a venis reforbentur, cum fangvine in circulum actæ in corporis nutrimentum et augmentum abeunt, dum rudiores in variis cavitatibus lecernuntur et tandem disfipantur. Edulia funt imprimis omnia gramina e clasfe Triandriæ, ut Cerealia, Secales Triticum, Hordeum, Avena, Oryza, Zea; omnes leguminofx e clasfe Diadelphiæ, imprimis Pifum, Pba 
feolus, Dolichos, Ervum, Vicia, Lathyrus, oleracere plan: tee ut Urrica, Spinacia, Beta, Chenopodium, Portulaca, Artocarpus, Brasfica culta; radices carnola Dauci, Paftinacæ, Rapæ et bulbofæe, ut Ixire, Gladioli, Diofcoxex; frucrus varii carnoli, ut Ficus, Dactylus, Ceraronia, Annone, Eugeniæ, Mufee, Cariç; nuces plurima ut Amygdala, Juglandes, Avellana, Fagi, Cocoes, et fexcenta alia.

\section{4 .}

Medicamina, ad forum medicum pertinentia, omnia et fingula ea funt fubfidia, ad quæ omnis æras, omne ævum luum habuit refugium in debellandis mcrbis, in reftituehda fanitate. Sub hac clasfe militant et militare debent remedia omnia, tam mitiora fua natura, quam fortio$\mathrm{ra}$, quæe proprie venena dicuntur, quæque prudenter adhibita vel refrecta dofi, vel diluta et mitigara fape morbos curarunt rebelles. Hæe remedia, pro qualirate diverfa, proque diverfa corporis vivi conftitutione varie admodum agunt. Robuftiori homini, firmiori fanitate gaudenti major plerumque dofis propinanda erit, dum laxioris compagis femina vix minimam ejusdem remedii portiunculam perferre poteft. Hæe eft ratio, cur rufticus robuftus integram Jalappa drachmam fine ulla noxa fumit, dum hyfterica mulier mitioris Rhei odorem absque vomitu et folutione alvi olfacere vix valet. Sic id ipfum remedium venenum huic cenferi poteft, guod illi falutiferum et innoxium pharmacum. Etjam infanti id esfe poteft venenum, quod adulto remedium, nifi quanticas cuilibet $æ$ tati præfcribenda, uti oportet, oblervatur. Mofchus odorem fpargit fexui mafculioo gratum, fequiori autem sexui, imprimis hyltericis, valde ingratum er ad deliquium animi ufque intolerabilem. Quxdam remedia nonnullis ani- 
malibus noxia funt, dum aliis vel omnino non, vel parum nocere folent. Animalia e Ferarum clasfe, quae cæca na. fcuntur, Canis, Lupus, Vulpes, Felis, Hyana facile omnia necantur Nuce Vomica et capiulis Euphorbice ac Toxicodendri, uti Sues pipere, Anferes perrofelino. Comeditur innocue Euphorbia ab ovibus et eapris, dum pecora exinde in Africa fepe fuccumbunt. Non femper omnes eiufdem planta partes aque funt noxiæ vel venenatæ; $n \geq c$ eadem pars, in una eademque planta, omni durationis rempore aque noxia obvenit: junior Phytolacca innocua comedi poreft absque noxa, aduitior velde corrofiva evadit, et deliciofa ficus matura, dulcisfima, nutriens, fi inma. tura ingurgitstur, fummopere acris er venenata vomitum ac diarrhoeam periculofam excitat. Obfervare quoque de. bemus, quod planta, fua natura venenata, præparatione ac cultura, edulis et falutaris retdi posfit, ut radix Ari, Nym. phrer, Solani tuberof, Campanulæ rapunculi, fructus Me. longenx:

\section{5 .}

Generaliter licet affirmari poteft, quod nnlla vere medicamina vel venena nutrientium naturam intra corpus. humanum acquirere posfint; multum tamen diu prudenterque continuato horum ufu effici poreft, ut paulifper hisce quafi asfuefiat natura, utque a minori ad majorem dofin fenfim adicendendo quandaque absque noxa ingurgitecur alexipharmacum, quod infverum fine dubio necaret. Confvetudo enim evadit quafi altera natura. Hæc eft ratio, cur tantam quantitatem venenati opii deglurire posfunt quotidie varize Aliam habitanies nationes: cur ufum ingrati non minus, quam venenati rabaci, omnes fere populi, nonnulli ad delirium usque amant: cur reme. dium, firpius repetirum e. g. laxars e Jalappa, Rheo vel Senna sandem inefficax evadit. 


\section{6.}

Hine concludi videtur, quod limites inter remedia et venena vix dentur alii, quam qui a dofi, xtate, confti tutione corporis, confuetudine et nonnullis aliis circum-d ftantiis dependeant. Multa antiquitus dicta venena, guæque interdum a Magiftratibus pro ufu medico, prohibita fuerunt, ut Antimonium, Mercurius, Arfenicum, quin et Opium, jam fape a cantis et peritis Medicis in ufum vocantur. Belladonna, Mandragora, Cicuta, pluraque alia Toxica, ab expertis Medicis, prudenti conflio prafcripta, pertinaces interdum morbos levarunt, arque vim medicatam exoptatam demonftrarunt.

Sint hæc de remediis, qua æquuilibrium funcrionum læfarum reftituunt, et de venenis quae viram tam vegetabjlem quam animalem deftruere valent, in genere dicta.

\section{70}

Venena, uti et remedia falutifera, e regno vegetabili plurima funt et valde varia.

Remedia audiant, qua mitius abfque periculo agunc, et venena proprie fint, qua periculola maxime funt et. vehementisfime vim fuam exferunt.

Ad remediorum clasfem referri posfunt:

r. Svaveolentia, ut Nyctanthes, Jafminum, quorum flores gratum fpergunt odorem et herba quamplurima e clasfe didynamiæ, oleo esfentiali fvavisfimo abundantes, imprimis Rosmarinus, Lavandula, Mentha, Ocymum, Teucrium, Thymus, Melisfa, Salvia: quæ omnes nervis noftris et cerebro amicisfimae fummopere excitantes et refolventes fint, in inflammationibus et morbis fogorofis ac paralyticis frepisfime ufitate. 
2. Arontata, quæ fimul calefacientia funt, ut Laurus, Piper, Myriftica, Coryophyllus, Illicium, Cinnamomum, plurimx ex umbellatarum ordine naturali et tantum non omnes cx ordine Ccitaminearum; imprimis Coriandrum, Anifum, Scandix, Coftus, Curcuma, Amoma omnia et Zingiber.

3. Amara, quæ humores acidos corrigunt et fibras mufculares corroborant, ut Cinchona, Ariftolochia, Qvasfia, Abfinthium, Carduus benedictus, Trifolium aquaticum.

4. Adftringentia, fortius fibras, quam Amara, firmantia et contrahentia, humores valde infpisfantia, optima nobis fubminiltrant remedia in profluviis tam alvinis, quam lan. guineis, ut diarrhoea, hæmorrhagiis internis et externis: qualia funt Sanguis Draconis, Cynomorium, Fraxinus, Quercus, Rofr, Stellatæ multæ.

5. Acida, fibras atcenuantia liumoresque imprimis fangvinis cruorem folventia ac refrigerantia, ut Pulpa Citri, Tamarindorum; fructus horæi ut Pyrus, Malus, Punica; Baccæe acido-dulces ut Berberidis, Vacciniorum, Vitis, Ribefiorum, Rubi, Mori et reliquorur.

\section{8.}

Hæ omnes materix cum non nutriant, nec corpus nutrire posfint, fed vâriis modis fanum lædant, morbofum ftatum mutent, veneni quidem nomen merentur, fed mitioris, ideoque utat protracto tantum ufu alterantia re. media falutari posfunt.

Ad fortiorum venenorum clasfem merito referimus:

6. Asria, quæ pro diverfo, quem in diverfis morbis 
et in diverfis corporis humani partibus monftrant effectum, varias acceperunt denominationes. Dinretica enim dicta fuerunt, dum in vias agunt urioæ. Intidentia, quum vifcidum omne facile folvant. Antifcorbutiva dum Scor. buto imprimis obicem ponune. Errlina, dum nervos ol. factus irritant et fternutationem excitant: Laxantia, dum modice alvum ducunt; Draftica, dum cum corminibus vehenenter contenta alvina expellunt. Rubeficiontia, dum curi externe applicata epidernidem leviter irritant. Epispaftica, dum fortius cutem corrodunt. Vificantia, dum fortisfime irritando cutem in veficulam elevant ac uberiorem lympba afBuxum causfant. Ejusmodi remedia non raro veneni acerrimi naturam induunt et faltim in. rerne caute adhibenda. Ad hanc clasfem referuntur plu. rimæe e clasfe Tetradynamix, ut Lepidium, Armoracia, Cochlearia, Raphanus, Brasilca, Sinapis et non paucæe e clasfe Polyandriae, fpeciarim Ranunculus, Atragene, Clematis, Adonis, Anemone, Piperitr: Lactefcentes ut Euphorbia, Elaterium, Gunni Gutra, Ricinus, Bi yonia: Con. torta ut Ipecacuanha, et Convolvulaceæ ut Jalappa, Scam. monum,

7. Anodyna, viræ animali imprimis inimica et, nifi caute adhibita, fummopere venenata, foporifera, apoplectica et letifera. In morbis dolorificis fenfum fopiunt; in fpafinodicis et convulfivis bæc prafcripes optima et certisfima fut remedia, ut in morbis mentalibus. $\mathrm{Mi}$ nori dofi doores et anxietates rollunt, exhilarant, inebrianr: majoris mentis operationes confundunt, memoriam fupendunt, infaniam et maniam excitant, mortem in ferre valent. Sic heroica maxime medicamina, nifi do feos ratio habeatur prudentisfima, venena hæc evadere posfuat maxime noxia et mortifera. Venenatam fuam 
naturam facile omnia monftrant eo, quod gufui non modo fint ingrara, fed quoad odorem imprimis naufeofa, ut Cannabis, Sambucus, Pxonia, A conitum, Digitalis, Fungi, Opium, præ aliis vero plantæ fic dictæ luridæ, ut Datura, Hyofcyəmus, Atropa, Solanum, Nicoriana. 


\section{DE PLANTIS VENENATIS}

VENIA. EXP. FACULT. MED. UPSÁt.

\section{PRASIDE}
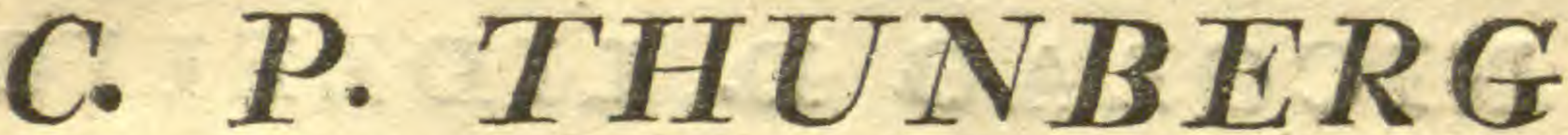

Commendatore Reg. Ord. de Wasa

Med. Doct. Prof, Med et Bot, Reg, et Ord. Reg. Coll. Sanit. Memb* Hon. Acad. Casar. Petrofnt. ei Nat. Curros. Reg. Scient. Ups, lond Hoim. Agric. Med. et Evang. Societ. Oecon. upsal. Patr. Hotm. ex. Civ. Oecon. Fint. Honor. Wermel. junec. Westm. Calm. et ỏrebr Berol ex lips. Nat. Scrur, Paris. Hagn. Moscou, et Halens. Hist, Nat. Gorknis. Philad. Lund, Harlem. Amsteld, Oecon, et Insti Litt. Ze-

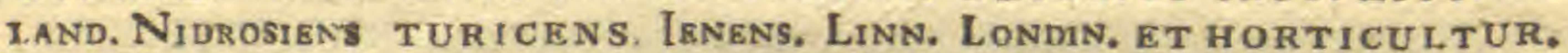
Reg. Scient. Pyys, et Phytc grapiy. Gótringens. Scientr. Gothot. Munach. Ertang. Wetter. Nanciens. Marpurg. Med. Paris. Emul. et Linn. Monspet. Med. Matritens, Med. et Nat, Studios, Enimburg, E? Petrop. Medico Chtr. et pharmac, Membronec non Acad. Scient. Paris, et Institut Nation, Monspgliens. Agricuit. Paris. Medic. Londiso Scandinav Florentin. et Batavin. Ind, Or. Correstond.

\section{PRO GRADU MEDICO}

$$
\text { P. P. }
$$

\section{ESTA NUS EM AN. HARLIN \\ Stip. Reg. Bothniens.}

IN AUDIT. BOT. DIE

JUNII MDCCCXXII

HORTS CONSUETIS

P. II.

U P S A I I

EXCUDEBANT REGI质 ACADEMIAE TYPOGRAPRI. 
RECTORI SCHOLF CELEBRATISSIMO, PR RCLARISSIMO DOMINO MAGISTRO

\section{INICORAO D. XIDEXIO}

A MICO OPTIMO

DIEATUM

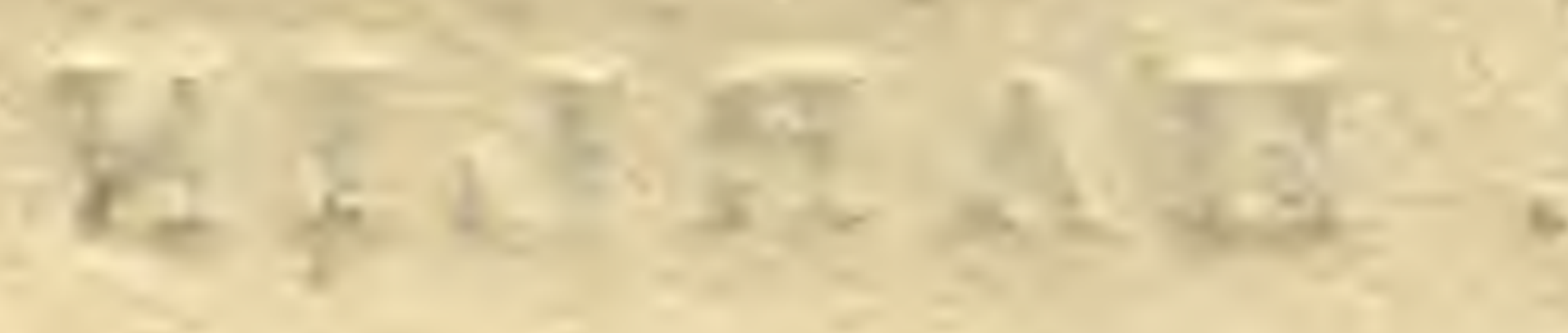

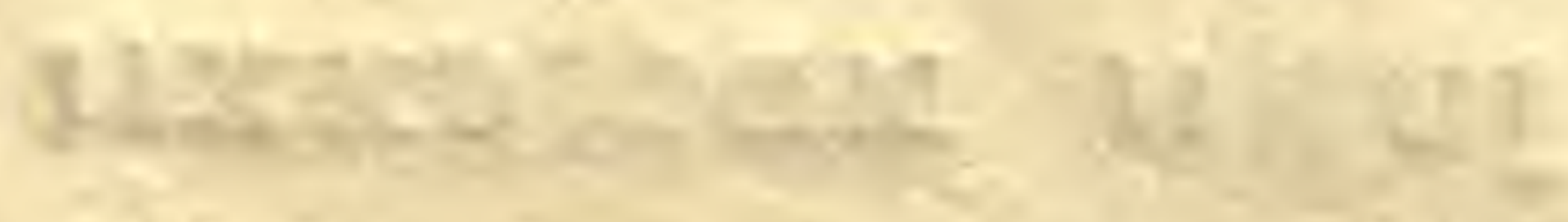

Voluit, debuit FET, HARLIN. 


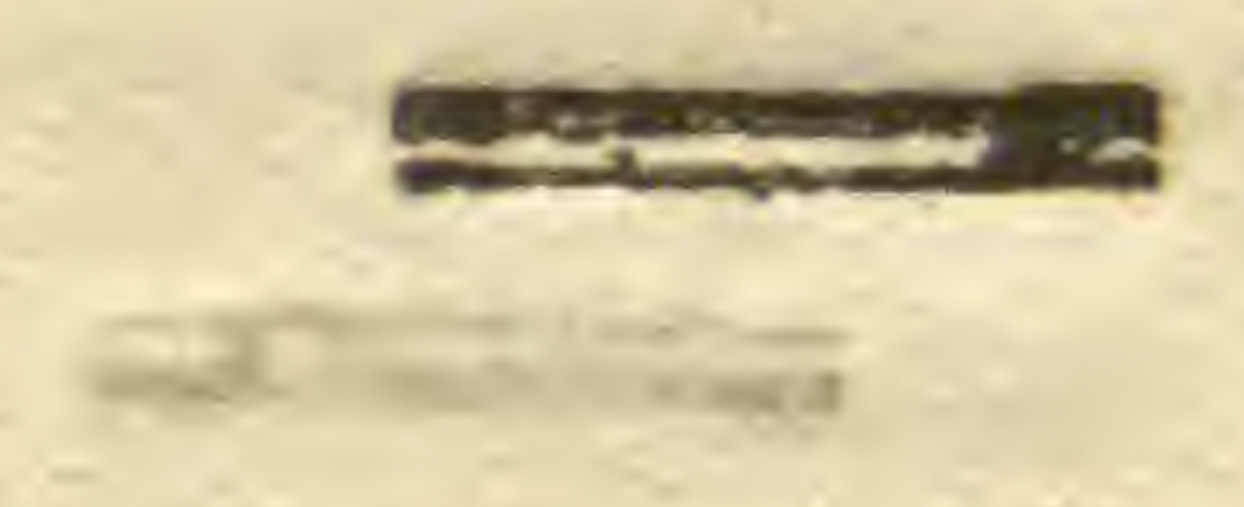

\%.

Venenatas plantas, quales ubique crefcentes, vel fpontaneæ, vel cultze occurrunt, aliter dignofcit peritis Botanicus, aliter rudis et indoetus homo.

Cum itague in regno vegetabili nonmullae clasfes et integri ordines naturales vel innocuas contineant plantas, vel noxias et valde venenatas. Botanicus bene eruditus facile edulia et utilia diftinguere valebit a remediis et venenis nocivis non modo lenfibus externis, fed etiam ex affinitate familiæe et qualitatibus plantæ proprits. Sic perbene fibi notum habet, guod omnia Gramina, qua omnes eo. rum partes, nutrimentum præbeant faluberrimum et omnium copiofisfinum tam animalibus phytivoris, quam homini, cujuscunque climatis incolae; guod pari modo Leguminofar, diadelphize clasfis ptantæe, tam caulrbus fữs fæpius fcandentibus, quam leguminibus fapidis nutriant hoininum animaliumque innumera millia; quod sitisfimæ Palma et medulla caudicis et numerofis fuis fructibus alimentum præbeant gratisfimum et falubre; quod Ico. fandria fpeciolæ arbores et trutices bacciferæ, uti fuperbiunt multitudine florum elegantium, ita etiam fructibus pomiferis et baccatis, carnofis et pulpofis, in ufum regni animalis multifarium abundent. Ubi ergo vel graminis genus culmo fiftulofo, articulato et femine nudo infructum: vel diadelphiam folits compofitis cirrhiferis, flore papilionaceo et legumine enfiformi, vel palmam caule indivifo foliisque terminalibus: vel fructum Icofandrum invenerit, numquam de utilitate ejus aut dubitat, aut frui illo vel minimum hafitat. 


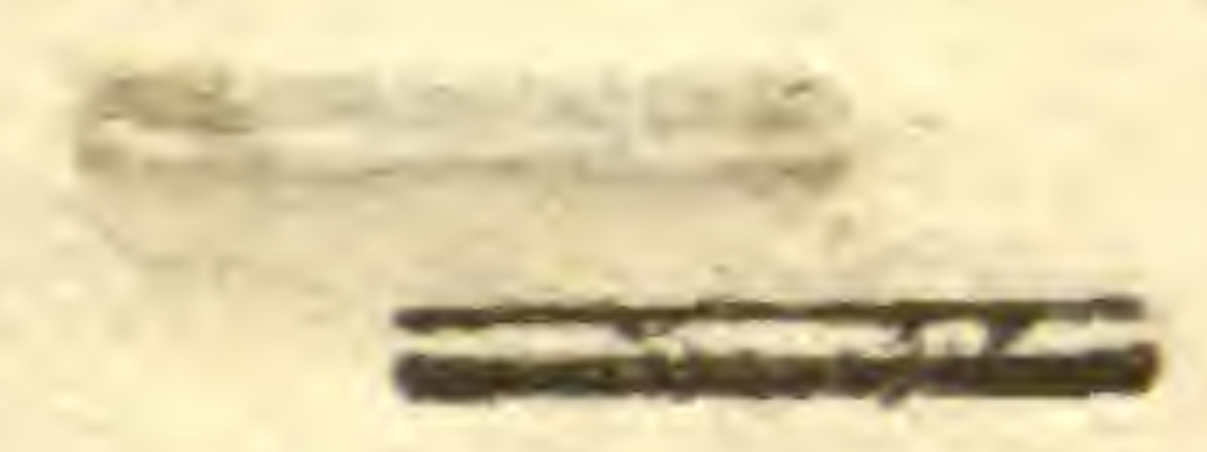

\section{5. ro.}

Facili quaque negotio Botanicus notas fibi et familiares reddit eas vegetabilium clasfes artificiales et naturales ordines, qui mitiora continent venena, quæque lub titulo amoeno medicaminum comprehendere folent artis medicæ Magiftri, humani generis dilecti fautores. Omnia igitur lvaveolentia, aromata, amara, adftringen. tia, acida, oleofa, cetera, illi ea funt exoptata et felicia arma, quibus non modo turbis, intra corpus bumanum ortis, obviam ire, fed et morbos leviores aque ac graviores non modo lenise, fed etiam radicitus tollere valet. E elasfe cruciatarum optima antifcorbutica, detergentia, diuretica, etiam epifpaftica prafcribit: e monadelphia plurima mucilaginofa : a coniferis balfamica et refinofa: e verticillatarum et ringentium ampla familia. Refolventia defumit ex Umbellatis flatulentia et ructatoria.

\section{11.}

Nec minus notæ Botanico erunt illæ clasfes et ordines plantarum, qui femper proferunt plantas venenatas, vel remedia periculofisfina nec fine cautela et circum. fpectione in morbis pertinacioribus adhibenda.

\section{Duplex horum præcipue eft genus:}

I- Acria, corrodentia et cauftica quoque dicta, Agunt hæe in folida corporis irritando, inflammando, ulcerando et deftruendo. Hujus efficacize funt planize plurimæ, numerofis ftaminibus germine infidentibus inftructa, Polyandræ dictæ; omnes lactefcentes et contortæ, quæ 
vomitum excitant et alvum laxant; omnes fructus tricocci ut Ricinus, Euphorbia, Croton, alii; piperitæ ut Arum, Dracontium, Calla. Ut taceam Mezereum, ficus immaturos, cucurbicaceas omnes.

2. Anodyna, qua in nervos agunt, functiones animales turbant, fopores excitant et vitam fupprimunt, diverfe admodum effectus fuos exferunt non modo pro diverfa zetate et conftitutione corporis, fed et pro diverfa fua qualitate, dofi et præparatione. Lurida pentandræ fingulari modo in cerebrum agunt, pupillam oculorum dilatant, fpasmos fedant, delirium excitant, oblivionem mirandam relinquunt. Fungi femper odoris ingrati, fæpe naufeof, funt interdum valde venenati, imprimis agaricus mulcarius, qui fymptomata, a plantis luridis non multum discrepantia, apud Berferkas aliasque nationes excitare folent. Opium e planta polyandra et finiul lactefcente naufeofum et ingratum guftui, quafi princeps inter narcotica refpici poteft. Protractior ejus ufus nervos debilitat, tremulentiam inducit, memorize vim minuit, et præmaturam fenectutem ac mortem accelerat, fpirituofa omnia diu immodiceque ufitata.

\section{12.}

Poftquam in antecedentibus paginis explicatum fuit; quid fit venenum? quomodo differat $a b$ efculentis et nutrientibus? Qua ratione venenum confiderari poteft vel mitius et minus periculofum adeogue remedii nomen mereri, vel fortius et fumme noxium, id eft mijori jure venenum dici oportet? Quibus adminiculis botanicie fcientize peritus et doctus Medicus plantas utiles a no: xiis, remedia a toxicis diftinguere valet? 
In fequentibus indicare conabimur, quomodo inda: ctus et omni notione plantarum fyftematica expers, facile et fecure plantas venenatas cognofeere posfit, quantum quidem id fieri poteft per pauca illa naturalia et fimplicia fubfidia, quibus rudis quisque homo inftructus esfe poteft, perque ilos facile inveniendos characteres, quibus plantas venenatas inftruere voluit omnipotens Creator, ut in tenfus hominis, parum oblervator is, cadec re posfent.

\section{13.}

Qualitates plantarum per analy fín chemicam eruere son pauci tentarunt Medici ut exploratis particulis conftituentibus earunque relativa proportione, eo melius: de viribus judicium ferrent. Hac vero via parum profecit fcientia, cum diverfæe plantæ, diverfa viriam indole pradita, multum in partibus conftituentibus invicem convenerint. Hæec methodus, fi vel magis proficua, quam. severa fit, foret, neque tamen facilis judicanda erit nes: fine multis impenfis adhiberi poteft; adeoque hoc plan. tarum chemicum examen indocto homini minirse commendandum erit.

\section{14}

Supereft igitur, ut indoles plantarum Fenemata psr illa fubfidia indagetur, quibus quemeamque hominem infruxit providens et alina natura, uti et bruta omnia ot fera animalia, quae ipfis bis, nec aliis adminiculis, ptilia eligere, noxia evitare perbene et fecure fciant.

Hac fubfidia funt fensus noftri externi optimi ill 
cuftodes, guibus guodlibet animalis genus iuftructum fuit, quique in hoc magis, in alio minus excellere folent$V_{i}$ fus rudis hominis parum vel nihil omnino conferre valet ad indagandas qualitates plantarum, cum notas, naturam illarum indicantes neque perfpieiat, neque intelligat. Nil itaque clasfificatio plantarum illi indocto detegere potef, nil facies externa ordinis naturalis; capfula tricocea nil illi fignificat; lac effuens veneni illi fufpicionem non femper excitat, bifi experientia edoctus nocivan ejus indolem expertus fuerit. Per tafum vix alias moxias plantarum fpecies evitare difcet, quam quæ vel aculeatze vel fpinofa armis fuis horrendis et laceranti: bus timorem illi injicere valent.

\section{6. $15:$}

Duos vero indubitatos; nec facile fallentes fenfus dedit benefica natura, quorum ope cuique facile erit indagatu, quid profit vel noceat, antequam per fauces ad tomachum pervenire posfit.

1:0 $N a$ us, artificiofe conftructas et plurimis inftractus nervis, per tunicam Schneiderianam disperfis, o. mne olidum per sëra volitans facillime excipit, five fit gratum, five ingratum. V'is hujus organi longe fortior apud feras nationes invenitur et in feris animelibus longe fortisfima, ut exempla demonftrant canum aliorumque. Cum itaque omni animali graveolentia, ambrofią ca, foetida et naufeofa maxime ingrata obveniant, fufpicionem facile noxil veneni excitant hæe, et de evitando periculo ferio admonent. Hajus ergo feolus ope facill 
negotio indigitantur venenatæ plantre luridæe et rarcoti. cæe, odorem fpargentes tetrum, idque adeo certe, indu bie, fecure et fubito, ut etiam hebetior nafus falli minime posfic. Pecora, glires et fera imprimis, quin et aves, omnia qua ing rate olent, evitare fciunt odoratu et utilia fibi edulia fecure eligere. Situs itaque nafi fupra os in omnibus animalibus naturali eft, et excellens organum olfactus indabie et fecure æque omne olidum noxium et venenatum fubolfacere poteft, ac celerrime inftinctu naturali oinne vivum cogere, ad id ipfum effagiendum. Vis graveolentium interdum adeo magna eft, bafo faltem emunctn, ut fyncopen et lipothymiam inde ortaın viderimus. Forti ideo fuo olfactu animalia, quorum nafus femper humidus deprebenditur, explorant follicite necesfaria alimenta, antequam guftui ea ulterius committunt examinanda.

3:do Lingua, organum faporis, papillis nerveis undi. que obfita, non minus facile, fubito, fecure et absque multis ambagibus omne id detegere valet, quod aliqua fcatet acrimonia et nociva ac corrodente qualitate vel quod naufeofum et nervis ingratum obvenit. Perfectior itaque hic guftus fenfus, quam olfactus, confiderari potelt, cum non folum acria, fed etiam narcotica detegere valet, et ad hæc refpuenda ac evitanda fortiter cogit. Animalia ideo, tam manfveta, quam imprimis fera a teneris usque annis hoc fenfu edocentur, plantas nocivas ab utilibus, venenatas ab efculentis difcernere et per totam deinde vitam effugere. Relinquunt itaque pecora in pratis acres Ranunculos intactos, ad rivulos virofam Cisutam evitare fciunt et fexcentas alias venenatas herbas angve pejus fugiunt. Si enim vitulus vel femel acrem guftaverit anemonem nemorofam, torminaque inde ven- 
tris contraxerit, vacca vel taurus numquam ulterius experiatentum illud iterabit.

Diftinguit facile omnis fana lingva aroma quodcum. que a naufeofo, acidum ab amaro, calidum a frigido, ficcum a ftyptico, dulce ab acri, oleofum a corrofivo, infipidum $a b$ omni falfo; et animalia inftinctu naturali, homo fapiens voluntate libera utens noxia omnia et fingula evitare posfunt facillime.

\section{$\$ 16$.}

Bini hi fenfus, præcipua quidem funt adminicula, quibus animalia, ad examinandas plantas venenatas utuntur, et quæ homini indocto primarii etiam erint $\mathrm{cu}$ ftodes, ne quid noxii facile ingurgitetur; fed homini, præcellenti præ brutis ingenio prædito adhuc alia patet via, qua ad certiorem, fi non certisfimam cognitionem de qualitatibus et viribus plantarum pervenire poteft, et qua in dubiis cafibus, quin etiam tentetur, prætermitti non debet. Scilicet dum quis, an planta vel fructus ejus noceat anxius et dubius hæeret, per animalia domeftica, acutioribus fenfibus inftructa, ejus effectus explorare poteft, eadem omnino ratione, qua Medici per canes venenata præparata artificialia extra dubitationis aleam ponere folent. Et hoc quidem experimentum non folum facile et fubito, fed etiam absque magnis impenfis, et fine temporis dispendio inftitui poteft, proficuum imprimis, quin et quandoque necesfarium peregrinatori prudenti, fub itineribus in regionibus incognitis, ubi omnia nova et ante non nota occurrunt.

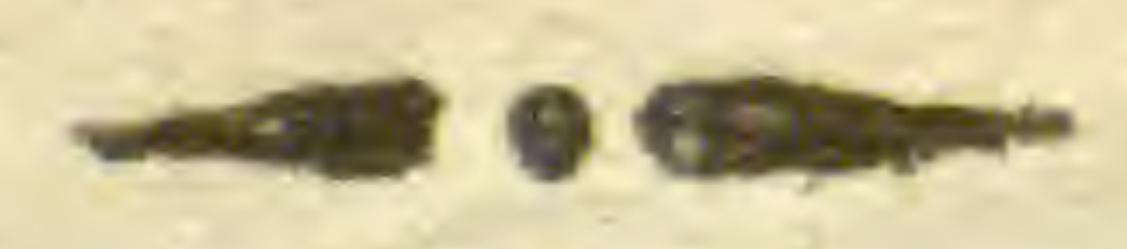

